\title{
Measurement of QT Interval and Duration of the QRS Complex at Different ECG Sampling Rates
}

\author{
MR Risk ${ }^{1}$, JS Bruno $^{1}$, M Llamedo Soria $^{1}$, PD Arini ${ }^{2}$, RAM Taborda $^{3}$ \\ ${ }^{1}$ Universidad Tecnológica Nacional, Buenos Aires, Argentina \\ ${ }^{2}$ Instituto Argentino de Matemática, Buenos Aires, Argentina \\ ${ }^{3}$ Universidad Nacional de Córdoba, Argentina
}

\begin{abstract}
The precise measurement of $Q T$ interval and $Q R S$ complex duration is important to characterize the electrical cardiac activity of the surface ECG. Variations of these intervals were evaluated when ECG sampling rate changes. The ECG recordings used in this study $(n=$ 78) were obtained from a combination of pharmacological blockage and postural changes. The ECG recordings were originally sampled at $500 \mathrm{~Hz}$, and then were downsampled by intervals of $25 \mathrm{~Hz}$ until the minimum sampling rate of $75 \mathrm{~Hz}$. In all cases the down-sampling was implemented using cubic spline interpolation. The QRS complex duration, RR and QT intervals for each subject, condition and sampling rate were measured. The QT interval was corrected using two different techniques: Bazzett and individualized QT corrections. The effect of the sampling rate was modeled with an exponential decay function, which was used later to measure where the exponential reaches an asymptotic value (at $5 \tau$ ). The $5 \tau$ value for uncorrected QT was at $290 \mathrm{~Hz}$, corrected QT with Bazzett was at $303 \mathrm{~Hz}$, and corrected QT with Individual method was at $253 \mathrm{~Hz}$, finally QRS complex duration was at 297 $\mathrm{Hz}$. An overestimation of the $Q T$ interval and $Q R S$ complex duration was observed when decreasing the sampling rate below $300 \mathrm{~Hz}$.
\end{abstract}

\section{Introduction}

The surface $E C G$ analysis is widely used for the diagnostic of cardiac diseases. The ECG is a noninvasive technique that allows the visualization of the heart's electrical activity. The changes in duration of the $Q T$ interval are related to certain pathologies, and with the effects of diverse drugs. On the other hand, the duration of $Q R S$ complex also gives information about the status of the myocardial conduction system. All this rise the necessity of making precise measurements of these intervals of the $E C G$ [1] [2].
The measurements on the $E C G$ are mostly defined by the characteristic extrema, amplitudes, wave morphologies and intervals of time between extrema points (Figure 1).

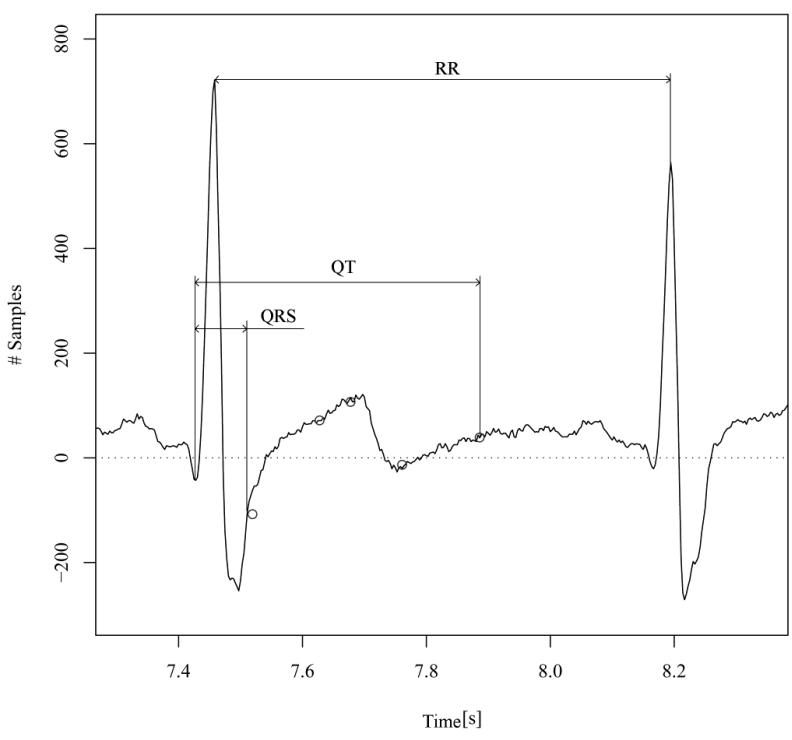

Figure 1. Definition of $R R, Q T$ and $Q R S$ complex in an ECG beat.

In the present study the $Q T$ interval and duration of the $Q R S$ complex were measured when the autonomic nervous system was blocked with a combination of drugs and postural changes.

Administration of atropine and propranolol was used to block sympathetic and vagal systems respectively, while subjects were in standing and supine position. This pharmacological and postural combination is useful to explore the $Q T$ interval correction techniques within a wide range of HR variations [3].

An incorrect choice of the sampling rate $\left(F_{s}\right)$ may introduce errors when the fiducial points of the $E C G$ are measured, and therefore the intervals or segments between 
these points.

The aim of the present study was to investigate the effects of the sampling rate in the measurement of $Q T$ interval and of $Q R S$ complex duration.

\section{Methods}

\subsection{Experimental protocol}

The database used in this work proceed from a previous work [3] where the regulation mechanism of the autonomic nervous system was studied. Electrocardiogram recordings were made on 13 adult subjects (age between 19 and 39, median 21 years). Those recordings were made during autonomic nervous system blockade by using pharmacological (atropine, propranolol) and postural (supine, standing) combinations.

The ECG recordings were made of the following way: a) all subjects were measured in supine control position $(S U C)$ then were moved to the standing control position $(S T C)$ and measured after 5 minutes for hemodynamic equilibration; b) then all subjects were returned to the supine position and given either atropine $(0.03 \mathrm{mg} / \mathrm{kg}$, $\mathrm{n}=7)$ or propranolol $(0.2 \mathrm{mg} / \mathrm{kg}, \mathrm{n}=6)$ reaching $S U A$ and $S U P$ condition respectively, both groups were measured after 10 minutes for equilibration; c) then all subjects were moved to the standing position, and after 5 minutes for hemodynamic equilibration STA and STP conditions were reached and measured respectively ; and d) finally subjects in $S U A$ and $S T A$ were supplied propranolol, and subjects in $S U P$ and $S T P$ were supplied atropine, in both cases this subjects reached total autonomic blockade $(S U B$ and $S T B$ ), and measured after 5 minutes. The database used in this work contains a total of $78 E C G$ recordings of 7 minutes each [3] [4].

\subsection{Acquisition and signal processing}

The ECG recordings were sampled originally at a frequency of $500 \mathrm{~Hz}$, then were down-sampled in steps of 25 $\mathrm{Hz}$ until a minimum frequency of $75 \mathrm{~Hz}$, therefore were obtained additional recordings at $F_{s}$ of 475, 450, 425, 400, $375,350,325,300,275,250,225,200,175,150,125,100$ and $75 \mathrm{~Hz}$.

In all cases, before changing the sampling frequency (resampling) an anti-alias filter was applied, defining the band of pass frequency $\left(F_{\text {pass }}\right)$, and a cutoff frequency $\left(F_{\text {stop }}\right)$ equal to half of the sampling rate. The specifications of the anti-alias filter were the following: a) equiripple FIR filter with a stop band of $40 \mathrm{~dB}$, b) bandpass ripple of $1 \mathrm{~dB}$, and c) $F_{\text {pass }}=80 \% F_{\text {stop }}$. The resampling was implemented with a cubic spline interpolation method [5].

The measurements of extrema points were made automatically with a program developed in $\mathrm{R}$ language [6]. The automatic measurement of the $Q T$ interval and the $Q R S$ complex, was preceded by the detection of the following extrema points: $\mathrm{Q}$ wave $(Q), \mathrm{S}$ wave $(S)$ and end of $\mathrm{T}$ wave $\left(T_{e}\right)$. The detection algorithm managed the presence of both monophasic and biphasic $\mathrm{T}$ waves, and either $P Q$ or TP segments may be used as the isoelectric baseline.

To ensure that the algorithms were applied correctly according to the $\mathrm{T}$ wave morphology and isoelectric baseline, a qualified observer classified the $E C G$ recordings, performing a randomly inspection of two minutes for each $E C G$ recording.

Once the detection of all the extrema points were completed, the $Q T$ interval and $Q R S$ complex were calculated. Only the heart beats with proper signal to noise ratio (SNR) and $R R_{[i]}>450 \mathrm{msec}$ (equal to a $H R<$ $133 \mathrm{bpm}$ ) and $R R_{[i]}>0.75 * R R_{[i-1]}$ were take it into account.

Since some $E C G$ recordings had high frequency noise, they were smoothed with a moving average filter (low pass, cutoff frequency of $10 \mathrm{~Hz}$, and 17 taps). This filter was designed to preserve the $T$ and $P$ waves [7]. Isoelectric baseline movement was removed from the $E C G$ after interpolating this movement with cubic spline method [5]. This procedure was repeated for all $F_{s}$.

Finally, the $Q T$ interval was corrected $\left(Q T_{c}\right)$ by two correction methods: Bazzett $\left(Q T_{c B}\right)$ [8] and Individual $\left(Q T_{c I}\right)$ [9]. Bazzett's correction uses the following parabolic expression:

$$
Q T_{c}=\frac{Q T}{R R^{\alpha}} \text { with } \alpha=0.5
$$

In the Bazzett's correction formula $R R$ is in seconds, and $\alpha$ is fixed at 0.5 . In the case of Individual correction the $\alpha$ value is calculated for each recording using a correlation model between $Q T$ and $R R$. This method uses the $\alpha$ value which correspond to the minimum value of the coefficient of determination $\left(r^{2}\right)$ between $Q T$ and $R R$. The figure 2 shows the diagram of the individual $Q T$ correction.

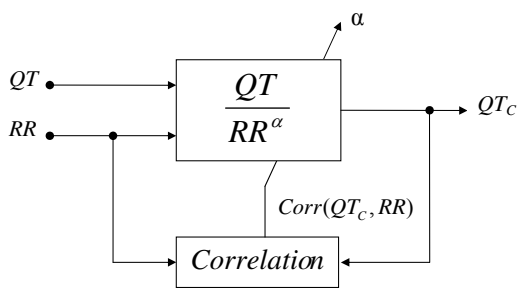

Figure 2. Diagram of the individual $Q T$ correction. 


\subsection{Modelization of sampling frequency changes}

The effect of the sampling rate was modeled with an exponential decay function:

$$
Y\left(F_{s}\right)=y_{0}+a \cdot e^{-F_{s} / \tau}
$$

From this model, the minimum sampling rate was calculated. At this frequency the exponential function reaches the asymptotic value, this value is reached at a frequency of approximately five times $\tau(5 \tau)$.

\subsection{Statistical analysis}

Analysis of variance (ANOVA) test were made for all variables under study, followed by a Bonferroni's post-hoc for multiple tests correction. All the statistical analysis were performed using the $\mathrm{R}$ language [6].

\section{Results}

The results are expressed as mean \pm tandard deviation. This value was obtained averaging the mean values for each $E C G$ recording. Table 1 summarizes the results.

The RR was statistically significant different in SUA $(P$ $<0.05)$ and SUB $(P<0.001)$ respect to control. On the other hand, differences statistically significant were found in STA $(P<0.05)$, STP $(P<0.005)$ and STB $(P=0.033)$ respect to control. The figures $3,4,5$ and 6 show uncorrected mean $Q T$, mean $Q T_{c B}$ by Bazzett and mean $Q T_{c I}$ by Individual correction. The exponential model is represented in solid lines. Mean value and standard deviation at both sides. The dashed lines correspond to the horizontal asymptotes, and each point corresponds to the mean of the variable under study. Vertical lines represent the standard error of each mean.

The $5 \tau$ frequency was at $290 \mathrm{~Hz}$ for $Q T$, at $303 \mathrm{~Hz}$ for $Q T_{c B}$, at $253 \mathrm{~Hz}$ for $Q T_{c I}$, and at $297 \mathrm{~Hz}$ for $Q R S$.

The measurement at 500 and $75 \mathrm{~Hz}$ was 408 and 423 ms for $Q T, 469$ and $485 \mathrm{~ms}$ for $Q T_{c B}, 447$ and $453 \mathrm{~ms}$ for $Q T_{c I}$, and finally 116 to $132 \mathrm{~ms}$ for $Q R S$ complex duration.

\section{Discussion}

The present study used a database where a wide variation range of $R R$ and $Q T$ intervals were obtained using combinations of pharmacological and postural procedures. The recordings in this database allowed the analysis of $R R$ and $Q T$ intervals in a full range of physiological situations, therefore it was possible to appreciate the effect of two $Q T$ interval correction methods. Finally, it was verified that sampling rate affects the measurements of $Q T$ interval and

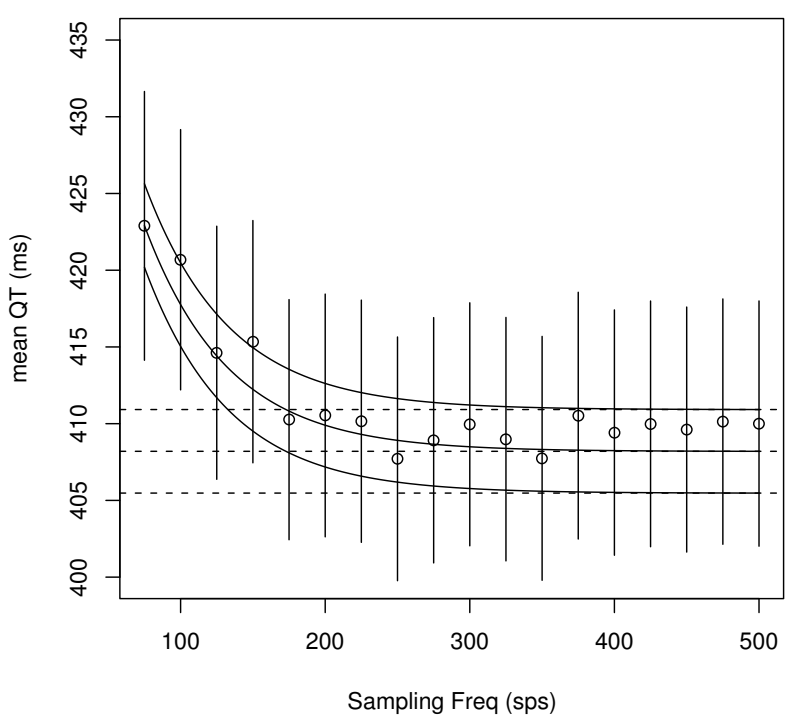

Figure 3. Mean $Q T$ model versus $F_{s}$.

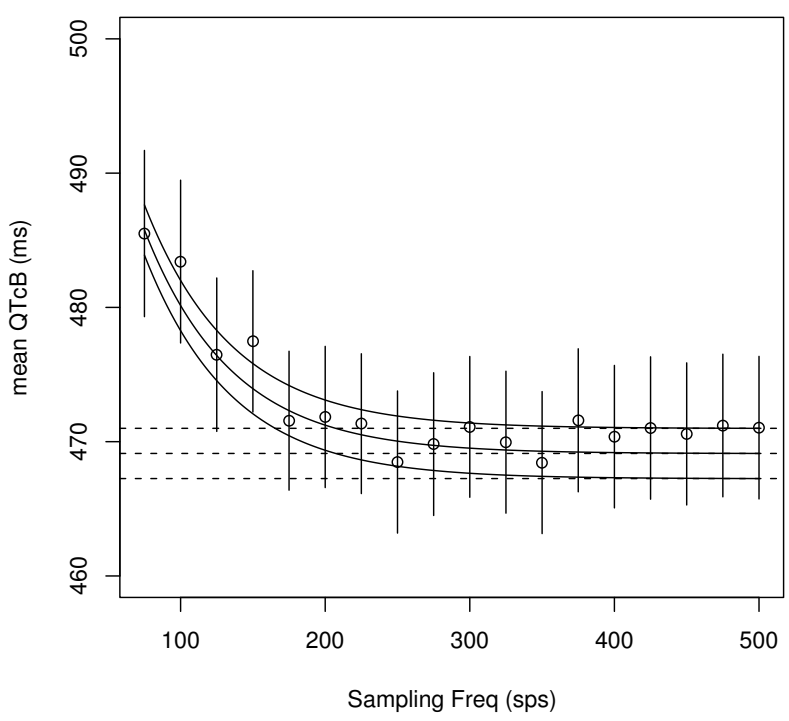

Figure 4. Mean $Q T_{c B}$ model against $F_{s}$.

duration of $Q R S$ complex, in both cases these variables were overestimated at $E C G$ sampling rates below $300 \mathrm{~Hz}$.

\section{Acknowledgements}

Authors thanks to AECI (Agencia Española de Cooperación Internacional) for the grant of the program "Proyectos Conjuntos de Investigación (PCI) IBEROAMÉRICA 2004". The present study was developed under the collaboration program between the Universidad Tecnológica Nacional, Argentina, and Universidad 
Table 1. Supine position, results in milliseconds as Mean \pm Standard Deviation.

\begin{tabular}{ccccc}
\hline Variable & Control & Atropine & Propranolol & Both \\
\hline$R R_{M} S u$ & $878.9 \pm 112.0$ & $604.4 \pm 139.2^{\ddagger}$ & $1038.7 \pm 144.8^{*}$ & $655.4 \pm 62.7^{\ddagger}$ \\
$R R_{M} S t$ & $738.8 \pm 96.3$ & $502.4 \pm 56.9^{\ddagger}$ & $912.2 \pm 88.1^{\ddagger}$ & $645.34 \pm 50.1^{*}$ \\
$R R_{S D} S u$ & $76.3 \pm 28.1$ & $19 \pm 26.2^{\ddagger}$ & $84.2 \pm 55.2$ & $11.5 \pm 7.7^{\ddagger}$ \\
$R R_{S D} S t$ & $72.4 \pm 37.9$ & $15.3 \pm 9.1^{\ddagger}$ & $67.2 \pm 54.3$ & $11.8 \pm 4.2^{*}$ \\
\hline
\end{tabular}

* $(P<0.05) ;{ }^{\dagger}(P<0.005) ;{ }^{\ddagger}(P<0.0005)$

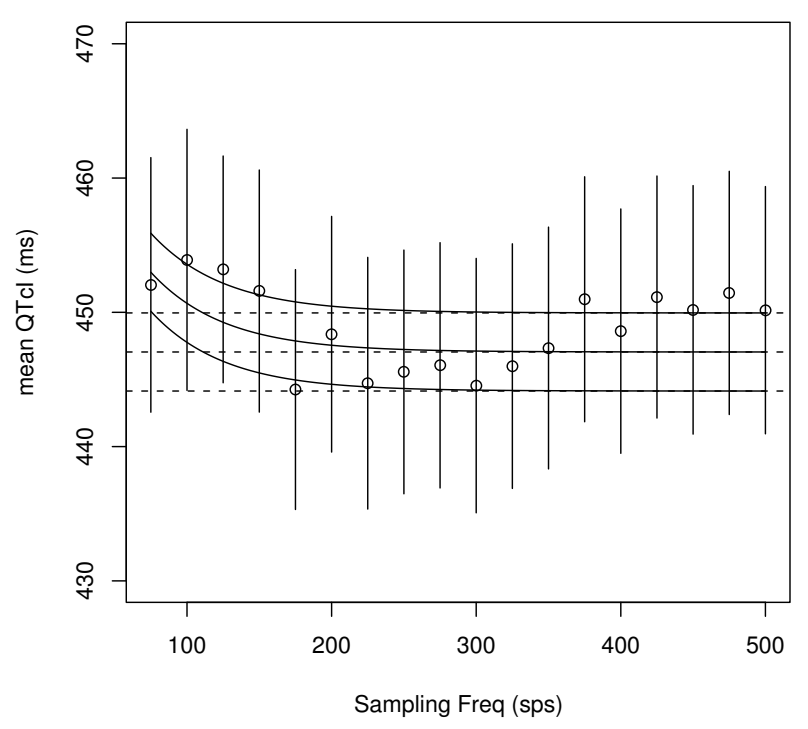

Figure 5. Mean $Q T_{c I}$ model against $F_{s}$.

de Zaragoza, Spain.

Authors thanks to Microsoft Research, Redmond WA, USA, for the donation of software and hardware where this study was performed.

\section{References}

[1] Ahnve S. Correction of the QT interval for heart rate: Review of different formulas and the use of the bazett's formula in myocardial infarction. Am J Cardiol 1985;109:568-574.

[2] Malik M. The imprecision in heart rate correction may lead to artificial observations of drug induced QT interval changes. PACE 2002;25:209-216.

[3] Saul J, Berger R, Albrecht P, Stein S, Chen M, Cohen R. Transfer function analysis of the circulation: Unique insights into cardiovascular regulation. Heart Circ Physiol 1991; 30:H1231-H1245.

[4] Risk M, Sobh J, Barbieri R, Saul J. A simple algorithm for QRS peak location: Use on long term ECG recordings from the HMS-MIT-FFMS database. In IEEE - EMBC, volume 1. 1995; 201-202.

[5] Meyer C, Keiser H. Electrocardiogram baseline noise estimation and removal using cubic splines and state-space com-

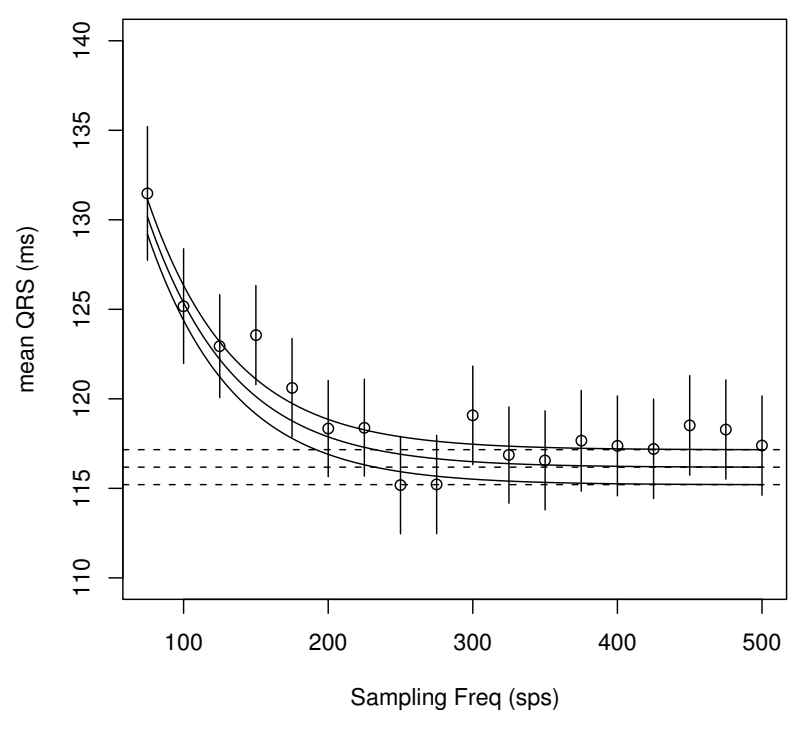

Figure 6. Mean $Q R S$ complex model against $F_{s}$.

putation techniques. Comput Biomed Res 1977;10(5):459470.

[6] Team RDC. R: A Language and Environment for Statistical Computing. Vienna, Austria: R Foundation for Statistical Computing, 2004.

[7] Tompkins W. Biomedical Digital Signal Processing, CLanguage examples and laboratory experiments for the IBM PC. New Jersey: Prentice Hall, 1999.

[8] Bazzett J. An analysis of time relations of electrocardiograms. Heart 1920;7:353-367.

[9] Smetana P, Batchvarov V, Hnatkova K, Camm A. Circadian rhythm of the corrected QT interval: Impact of different heart rate correction models. PACE 2003;26(Pt. II):383-386.

Address for correspondence:

Marcelo Risk, Ph.D.

Dpto de Electrónica - UTN FRBA

Medrano 952, Ciudad de Buenos Aires, Argentina marcelorisk@gmail.com 